Revue interdisciplinaire sur la Grèce archaïque

\title{
Les dieux d'en haut et les dieux d'en bas chez Homère : une question à réexaminer
}

Gods of the Above, Gods of the Below in Homer: A Question to Be Re-Examined

\section{Stella Georgoudi}

\section{OpenEdition}

\section{Journals}

Édition électronique

URL : https://journals.openedition.org/gaia/2364

DOI : 10.4000/gaia.2364

ISSN : 2275-4776

Éditeur

UGA Éditions/Université Grenoble Alpes

Édition imprimée

ISBN : 978-2-37747-292-5

ISSN : $1287-3349$

Référence électronique

Stella Georgoudi, « Les dieux d'en haut et les dieux d'en bas chez Homère : une question à

réexaminer », Gaia [En ligne], 24 | 2021, mis en ligne le 31 mai 2021, consulté le 09 décembre 2021.

URL : http://journals.openedition.org/gaia/2364 ; DOI : https://doi.org/10.4000/gaia.2364

Ce document a été généré automatiquement le 9 décembre 2021.

Gaia. Revue interdisciplinaire sur la Grèce archaïque 


\section{Les dieux d'en haut et les dieux d'en bas chez Homère : une question à réexaminer}

Gods of the Above, Gods of the Below in Homer: A Question to Be Re-Examined

Stella Georgoudi

\section{Introduction}

1 Pour introduire ce sujet, j'ai choisi une qualification des dieux plutôt générale et neutre, à savoir dieux d'en haut et dieux d'en bas ${ }^{1}$, cela pour échapper à une opposition bien connue, maintes fois utilisée par plusieurs Modernes, qui se plaisent à classer nettement les différentes puissances divines en deux groupes séparés : «olympiennes » (ou, plus rarement, « ouraniennes ») et « chthoniennes ». Parfois, on va même plus loin, en faisant la distinction entre " religion olympienne » et " religion chthonienne », ou entre « sacrifice olympien » et " sacrifice chthonien », ce qui conduit à établir ainsi une sorte de dichotomie rigide entre un rituel adressé aux soi-disant «Olympiens / Ouraniens » et d'autres rites qui auraient concerné seulement des entités cataloguées comme « chthoniennes ", qu'il s'agisse de dieux ou de héros.

Ce postulat a été forgé dès le début $\mathrm{du}$ xIx ${ }^{\mathrm{e}}$ siècle et, malgré les critiques qui ont commencé à voir le jour surtout depuis le milieu du $\mathrm{xx}^{\mathrm{e}}$ siècle, ce type d'opposition continue à être défendu par des spécialistes de la Grèce ancienne. Certes, en règle générale, nous pouvons dire que certaines puissances ont des relations plus étroites avec le monde visible, avec ses hauteurs et sa voûte céleste, que d'autres qui sont beaucoup plus associées au monde d'en bas. Si l'on restait sur cette constatation générale, tout le monde pourrait être d'accord. Les choses se compliquent lorsqu'on veut ériger cette constatation en un axiome, en une sorte de schéma archétypal à travers lequel on pourrait saisir l'essence de la religion grecque. De ce point de vue, ce qu'écrit William Guthrie, fervent partisan de cette conception, est parfaitement révélateur : "The distinction between Olympian and chthonian, aetherial and sub-aetherial, or 
to put it more simply, between gods of the heaven and gods of the earth, is one which I hold to be fundamental for the understanding of Greek religion ${ }^{2}$. " Il faut cependant noter que, dans ce type d'écrits et de discours, le mot «terre » renvoie presque toujours au monde d'en bas.

3 Je ne vais pas faire ici, même brièvement, l'historiographie de ces théories qui ont été souvent répétées d'un auteur à l'autre, de telle sorte qu'elles sont apparues à certains chercheurs comme une évidence qui n'a pas besoin d'être prouvée. Je m'en tiens à renvoyer aux travaux éclairants de Renate Schlesier ${ }^{3}$, en soulignant seulement avec elle un autre aspect significatif qui marque ces théories. La dichotomie "Olympiens / Chthoniens » est, en effet, liée parfois à une conception évolutionniste de l'histoire, selon laquelle, le « culte chthonien » appartiendrait à une première étape du développement de la religion grecque, tandis que le "culte olympien" représenterait une phase postérieure. Or, pour Jane Harrison, qui s'appuie surtout sur les théories développées par Edward B. Tylor, ainsi que par Johann Jakob Bachofen (dans son Mutterrecht [Le droit maternel]), cette transition de la religion chthonienne à la religion olympienne aurait coïncidé avec la transition du matriarcat au patriarcat ${ }^{4}$. Elle dresse ainsi une sorte de schéma structural établissant une distinction entre, d'une part, l'élément "chthonien », associé au plus ancien, au féminin, au religieux, au local, et, d'autre part, l'élément « olympien », lié au plus récent, au masculin, au panhellénique ${ }^{5}$. Une évolution analogue sera dépeinte par Erwin Rohde, bien qu'il ne trouve pas de preuves en Grèce d'un "ancient system of "matriarchy" ». Selon ce grand savant, les dieux d'Homère sont "pan-Hellenic, Olympian", mais avant lui, les "chthonic deities [...] are among the oldest possessions of Greek religious faith ». Cependant, il y aurait ensuite un retour en arrière. Car, après ce qu'il appelle "l'époque homérique », le culte des dieux "chthoniens " ainsi que le culte des morts seraient revenus en force. En considérant les «dieux olympiens » comme des êtres éloignés, distants, loin de leurs préoccupations, les Grecs se seraient surtout tournés vers les dieux locaux liés à la terre et au monde d'en bas, parce que ces dieux-là leur seraient apparus plus proches. C'est pourquoi Rohde considère que le "culte chthonien" a constitué la base des pratiques religieuses grecques, aussi bien pour les individus que pour les communautés civiques ${ }^{6}$.

Il est vrai que, depuis quelques années, certains hellénistes critiquent cette dichotomie, considérant qu'elle ne correspond pas à la polysémie, la variabilité, la plasticité des croyances et des pratiques des Grecs ${ }^{7}$. La discussion donc n'est pas close : on continue à reprendre et à reconsidérer ces deux catégories, on propose même de remplacer la distinction "Olympiens / Chthoniens » par leur " mélange ${ }^{8}$ ». Cependant, il n'en reste pas moins qu'on n'arrive pas toujours à se libérer des clichés et des idées reçues, afin d'essayer d'affronter l'extrême complexité des faits grecs, qui, parfois même, peuvent présenter des aspects contradictoires, dus entre autres à la nature et à la datation des sources que nous possédons. Ainsi, malgré certaines avancées dans le réexamen de cette dichotomie, on peut la retrouver, formulée dans toute son orthodoxie, dans un manuel récent sur la religion grecque ${ }^{9}$ : les divinités "ouraniennes» («Ouranic deities»), qu'on identifie souvent aux "olympiennes», seraient associées au ciel, au jour, aux autels (bômoi), au vin, aux repas, à des animaux de couleur blanche ou claire, tandis que les divinités " chthoniennes » (" chthonic deities ») seraient liées à la terre, à la nuit, à des autels bas et creux (escharai), aux libations sans vin (nêphalia), à l'absence de repas (ou à des holocaustes, surtout d'un porcelet), à des animaux de couleur noire. Cependant, ces oppositions binaires et rigides ne correspondent pas à la réalité cultuelle des Grecs, comme le montrent de nombreuses sources littéraires et 
épigraphiques, une réalité beaucoup plus diversifiée, d'une flexibilité et d'une plasticité certaine $^{10}$.

5 Toutefois, bien que - comme je l'ai dit - je n'aie pas l'intention d'entrer dans la problématique du « binôme » «Olympiens / Chthoniens », j'aimerais, avant d'aller plus loin, prendre un exemple - parmi d'autres - susceptible de montrer justement la complexité de nos sources. En cherchant des données "for the reality of the distinction " entre «Olympiens » et "Chthoniens ", tout en nuançant ses propos, Scullion cite un passage des Lois de Platon, où le philosophe aurait mentionné « five classes of divinities to whom honours are to be given sequentially: Olympians, chthonians, daimones, heros, and "private images of paternal gods" »"1. Mais que dit exactement Platon? Il énumère les honneurs qu'on doit rendre, dans l'ordre, aux " Olympiens ", aux « dieux qui possèdent la cité » et aux dieux « chthoniens ». Ensuite, " après ces dieux », on doit, dans l'ordre aussi, honorer les daimones, les héros, les dieux ancestraux et, enfin, les parents ${ }^{12}$. Si l'on comprend bien ce passage platonicien, on pourra remarquer, tout d'abord, qu'entre les Olympiens et les chthoniens, Platon place les dieux de la cité, ces poliouchoi theoi, qu'on ne saurait ignorer, comme le fait Scullion. En deuxième lieu, les daimones et les héros ne semblent pas être qualifiés de "divinités ", comme le suggère le même auteur, en les englobant dans les "five classes of divinities"; Platon les mentionne

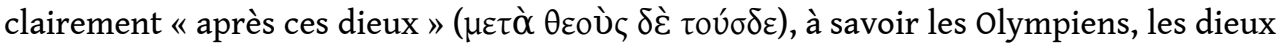
de la cité et les chthoniens. Enfin, dans cette énumération, je ne pense pas que Platon mette l'accent sur une "distinction» entre Olympiens et chthoniens, deux « ensembles » qui ne sont même pas cités côte à côte; les uns et les autres font tout simplement partie d'une série d'instances, divines, héroïques ou autres, que les hommes doivent honorer pour témoigner de leur piété, de leur eusebeia.

\section{La terre homérique (chthôn, gaia) et le monde d'en bas}

6 Maintenant, si l'on veut sortir de ces généralités ou approximations, éviter de penser par oppositions tranchées, saisir la façon dont les Grecs eux-mêmes concevaient leurs dieux, il serait utile, voire indispensable, de scruter attentivement le vocabulaire, le sens des mots utilisés pour qualifier une divinité, un espace, un mode d'action - ce qui paraît, certes, une évidence, mais une évidence qui est souvent oubliée. Ainsi, l'examen contextuel de certaines épithètes comme chthonios, olumpios, mais aussi ouranios, est une condition préalable à tout classement des divinités grecques. Par exemple, pour plusieurs des Modernes, un dieu chthonios serait surtout un dieu "infernal», "souterrain", associé au monde d'en bas ${ }^{13}$. Cependant, comme on sait, cette épithète renvoie au terme chthôn, " terre ", un espace qu'on pourrait, à première vue, diviser en deux parties : une surface étendue, foulée par les humains, et un territoire qu'on laboure, une matière cultivable, caractérisée non seulement par l'horizontalité, mais aussi par la verticalité, puisque la semence est enfouie dans cette chthôn ${ }^{14}$. Toutefois, le type de profondeur de cette terre qu'on laboure et qu'on cultive n'est pas le même que celui des profondeurs qu'implique le monde infernal. Ainsi, en règle générale, le Zeus Chthonios n'est pas un dieu "infernal ", comme on le traduit souvent, il n'est pas un Zeus Katachthonios, comme nous allons voir. Les deux, en effet, ne sont pas obligatoirement interchangeables. Le laboureur d'Hésiode qui, dans les Les Travaux et les Jours, prie Zeus Chthonios et la pure, la chaste, l'hagnê Déméter, n'adresse pas sa prière à des divinités 
infernales, mais à des puissances divines associées aux travaux des champs, qui sont susceptibles de veiller sur la maturité des fruits de la terre ${ }^{15}$. Autre exemple : le Zeus Chthonios, qu'on trouve, beaucoup plus tard, dans un important calendrier sacrificiel de Myconos $^{16}$, n'a rien à voir avec le monde d'en bas. En effet, ce calendrier prescrit une série de sacrifices, accomplis dans un contexte agricole où, le 12 du mois de Lênaiôn

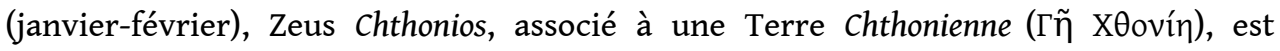

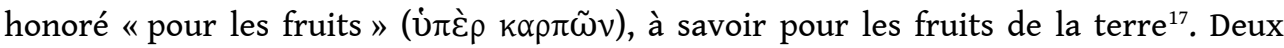
jours plus tôt, le $10 \mathrm{du}$ mois, "au moment du chant pour le fruit de la terre"

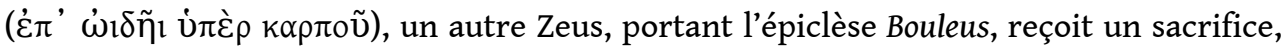
à côté cette fois de Déméter et de Korè (1.15-17). Or ces divinités sont évoquées, également ici, comme des puissances divines présidant aux travaux agricoles, dans un contexte qui concerne l'agriculture, la fécondité, le souci des karpoi; il n'y a rien qui puisse suggérer ici une relation avec la mort et le monde d'en bas ${ }^{18}$. Par ailleurs, le fait de sacrifier à Zeus Chthonios et à Gê Chthoniê des animaux « noirs écorchables» (1. 25 :

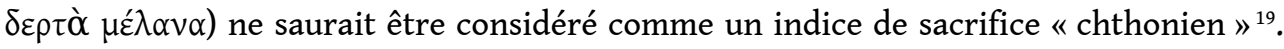
Le cas de Myconos montre que des animaux sacrificiels de couleur noire peuvent très bien être immolés à des divinités associées à la fertilité des champs. D'ailleurs, la terre,

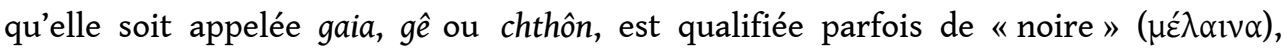
comme dans le fameux fragment de Solon, maintes fois commenté, qui parle de la "Terre noire » (Gê melaina), terre de l'Attique, que Solon avait libérée en arrachant les bornes qui y avaient été enfoncées partout. Certes, Solon divinise ici cette terre,

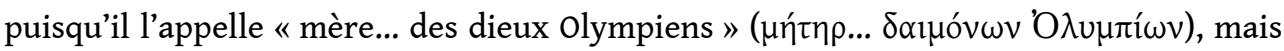
elle ne constitue pas moins cet espace qu'on cultive, et qu'il fallait libérer de l'esclavage en abolissant les dettes ${ }^{20}$. Or, en qualifiant cette terre de melaina, on renvoie sans doute aux tons sombres, presque noirs, qui caractérisent une terre de culture, une terre fertile, pleine de promesses pour une abondance de fruits ${ }^{21}$. On pourrait dire enfin, sans trop se tromper, que le Zeus Chthonios de Myconos est comparable au Zeus Chthonios dans la prière du laboureur d'Hésiode. Et j'ajouterai que Gê, en tant que divinité ici, a besoin même de l'épithète Chthoniê pour montrer son lien avec la chthôn, cette terre qu'on cultive et dont on espère avoir de bonnes récoltes.

7 J'aimerais cependant préciser, avant d'aller plus loin, que quand je mets en doute la traduction du terme chthonios par "infernal» ou par "souterrain", je ne veux nullement dire que chthonios ne signifie jamais infernal ou «appartenant au monde d'en bas ». La preuve, un passage de la Théogonie hésiodique, où Hadès est qualifié de chthonios theos (v. 767), et en ce cas cette épithète renverrait à l'espace d'en bas et deviendrait ainsi synonyme des adjectifs hupochthonios ou katachthonios, qui désignent ce qui est "sous terre ». De façon comparable, Sophocle qualifie d'ailleurs Hadès de "dieu d'en bas ", tandis qu'il appelle Perséphone « la déesse souterraine ». Et l'on peut donner éventuellement le même sens à un fragment d'un drame non identifié $\mathrm{d}^{\prime}$ 'Euripide, où il est question de theoi chthonioi ${ }^{22}$. On prend donc acte de tous ces cas et de ces ambiguïtés. Il n'en reste pas moins qu'on ne peut non plus, dans tous les cas et dans tous les témoignages des anciens, donner au mot chthonios une seule signification, celle qui l'associe au monde infernal et à ses puissances, comme on le fait parfois de façon presque automatique.

Venons-en maintenant à Homère pour voir si et comment ces soi-disant "catégories " de « dieux olympiens » (ou, plus rarement, « ouraniens ») et de "dieux chthoniens », se présentent. Une telle recherche doit prendre en compte toute une série de termes 
portant sur la terre et le monde d'Hadès, sur l'olympe et le ciel, sans oublier le Tartare $^{23}$, mais aussi ce qu'on désigne comme l'éther. Je précise tout de suite que l'examen complet et approfondi de ce vaste vocabulaire dépasse et de loin le cadre de cet article qui se limite à la recherche de certains exemple indicatifs, susceptibles de donner un premier aperçu de la question. Or, une approche même incomplète de ce riche vocabulaire montre tout d'abord l'absence du mot chthonios dans les poèmes homériques. Il est, certes, question parfois d'epichthonioi, terme qui désigne les hommes, les mortels (brotoi), qui vivent sur terre ${ }^{24}$. Mais on trouve surtout le substantif chthôn, « terre ", ainsi que l'autre mot signifiant la « terre ", à savoir gaia. Car c'est sous cette forme, gaia, que la terre est mentionnée chez Homère, tandis que le mot gê est très rare : je note seulement deux passages de l'Iliade où apparaît Gê, mais il s'agit alors de la divinité Terre. Au chant III, 103-104, il est question d'agneaux qu'on sacrifie à la Terre, au Soleil et à Zeus pour conclure un pacte, tandis qu'au chant XIX, 258-261, Agamemnon jure qu'il n'a jamais porté la main sur la jeune Briséis et en prend à

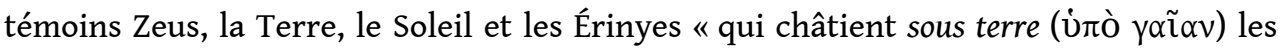
hommes parjures à un serment ». Or, on voit que c'est la forme gaia, et non pas gê, qu'on trouve ici lorsqu'on se réfère à la terre, en tant qu'espace cosmique.

9 Cependant, s'il n'y a pas de terme « chthonien » chez Homère, comment parler de dieux "chthoniens", dans le sens de dieux « infernaux», comme on continue parfois à le faire? On pourrait objecter que s'il n'y a pas l'épithète chthonios, il y a bien chthôn, la terre - qui peut se dire aussi gaia - et, dans ce cas, cette terre peut bien se référer, dans certains contextes, au monde d'en bas. Le problème c'est que, parmi tous les passages mentionnant chthôn ou gaia, aucun n'associe ces deux termes au sens « infernal ». Prenons tout d'abord chthôn. Dans l'Iliade et l'Odyssée cette chthôn désigne la terre en tant que cette "large » surface (eureia), " aux larges routes» (euruodeiê), foulée par les "races humaines" (phula anthrôpôn) ${ }^{25}$. Elle est très souvent qualifiée de pouluboteira, "celle qui nourrit beaucoup ", et elle apparaît, le cas échéant, comme kelainê, "sombre, noire ", sur laquelle Zeus déverse avec violence l'eau (l'hudôr) pour manifester sa colère aux mortels quand ils prononcent des sentences torses et ne respectent pas les dieux ${ }^{26}$. La chthôn est même qualifiée de dia, "divine ", car c'est elle qui fait naître un tendre gazon, du lotus frais, du safran et de la jacinthe, en créant ainsi un tapis serré et doux sur lequel vont s'étendre Zeus et Héra pour faire l'amour ${ }^{27}$. Enfin, les mortels (brotoi) habitent (naietaousi) sur la chthôn, ils mangent leur pain epi chthoni, ils tombent pendant la bataille sur cette terre, morts ou blessés, ou encore c'est cette chthôn qu'Héra prend dans sa main lorsqu'elle prête serment devant Hypnos ${ }^{28}$. Entre la chthôn et le ciel, il y a une grande distance que seule une puissance surhumaine peut combler : en plein combat entre les Achéens et les Troyens, Éris, la sœur et compagne d'Arès, se dresse, d'abord petite, puis elle grandit de plus en plus de sorte que sa tête touche fortement le ciel, tandis que ses pieds marchent sur la chthôn lorsqu'elle va et vient à travers les combattants pour jeter au milieu de tous l'esprit de la querelle ${ }^{29}$. Dernière touche au tableau: lorsque quelqu'un meurt, il est enterré "sous la terre", hupo chthonos, tandis que son âme s'enfuit comme la fumée « dans la terre, au fond de la terre » (kata chthonos), pour regagner le monde d'en bas, la demeure d'Hadès ${ }^{30}$.

Si l'on relève maintenant les passages concernant le terme gaia, on voit que ce mot est beaucoup plus mentionné que chthôn, mais avec des sens souvent comparables, même si l'on trouve aussi des formules particulières, comme on le verra, qui portent seulement sur gaia. La gaia homérique est une grande "nourricière» (poluphorbos), elle est également «immense", «infinie » (apeiresiê, apeirôn), elle résonne sous les pieds des 
guerriers, elle est imprégnée de leur sang qui la fait rougir, c'est elle qu'on " agrippe, du creux de sa main, en tombant dans la poussière $»^{31}$. Elle peut être qualifiée d'«insensible» (kôphê) ; mais une grande fureur, comme celle d'Achille, arrive à outrager cette gaia, lorsque le héros venge son ami Patrocle en attachant le cadavre d'Hector à son char et en le traînant sur le sol ${ }^{32}$. Comme dans le cas de chthôn, ceux qui meurent vont, "descendent ", "sous la terre " (hupo gaian, gaian edutên), et cette gaia, qualifiée d'« épandue » (chutê), on l'amoncèle pour qu'elle "recouvre» (kaluptei) ainsi les morts ${ }^{33}$. La gaia peut, par ailleurs, s'ouvrir par l'action d'un dieu très puissant (kreiôn), à savoir Poséidon, l'« Ébranleur de la terre»(Enosichthôn) ${ }^{34}$. Poséidon est capable de faire éclater ( $\dot{\alpha} v \alpha \rho \rho \eta ́ \xi \varepsilon 1 \varepsilon)$ la gaia, en ouvrant ainsi aux yeux des mortels et des immortels l'effroyable espace humide de la mort, que même les dieux ont en

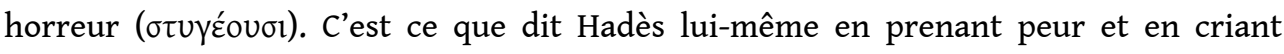
contre son frère Poséidon, quand ce dieu secoua ( $\left.\varepsilon_{\tau i ́ v \alpha \xi \xi}\right)$ la terre et les hautes cimes des montagnes et ébranla la cité des Troyens et la flotte des Achéens ${ }^{35}$.

On pourrait donc dire que la gaia homérique, pas plus que la chthôn, ne renvoie pas au monde d'en bas, même si cette terre peut aussi être conçue comme une matière, un espace doté d'une certaine épaisseur, comme nous l'avons vu. Par ailleurs la gaia peut aussi désigner la terre ferme, celle qu'Ulysse aperçoit, selon les moments, de très loin ou de près, comme un lieu de refuge (phuximon), en se demandant parfois auprès de quels mortels il revient ${ }^{36}$. Mais le mot gaia prend aussi un autre sens chez Homère. Il peut signifier le pays, la "terre des pères ", la patrie chérie, aimée (philê), qu'on désire ardemment atteindre lorsqu'on est loin d'elle. La formule patrida gaian ou philên es patrida gaian revient souvent dans les poèmes homériques, surtout dans l'odyssée ${ }^{37}$. Il est significatif que cette notion de "terre patrie » s'étoffe encore plus après Homère. Chez les Tragiques ou chez d'autres auteurs, elle peut être désignée de surcroît comme "mère » (mêtêr) ou/et comme "nourrice " (trophos), pour renforcer justement le côté maternel et nourricier du pays natal ${ }^{38}$.

\section{Zeus Katachthonios}

12 Revenons maintenant à l'appellation dieux "chthoniens " susceptible de se trouver aussi dans les poèmes homériques, selon certains savants. Le fait est qu'on n'y trouve pas de dieux qualifiés de "chthoniens", de chthonioi, pour la simple raison que cet adjectif est absent chez Homère (voir supra). Reste bien évidemment Hadès, qualifié d'« abominable ", d'« odieux » (stugeros), mais également de klutopôlos, « aux coursiers renommés », ce dieu «fort» (iphthimos), qui vit, avec la «redoutable» (epainê) Perséphone dans le monde d'en bas ${ }^{39}$. En effet, sa demeure, sa maison (domos, dôma, dô) " aux larges portes $»^{40}$, «solidement closes » comme le suggère l'adjectif pulartês qui caractérise le dieu, se trouve dans les "régions souterraines ", dans les profondeurs de la terre (hupo keuthesi gaiês) $)^{41}$. C'est dans cette demeure que les morts seront « envoyés " (pempô), c'est là où ils vont «aller » (bainô), où ils vont "descendre » (katerchomai), où ils vont être "renfermés » (keuthomai) ${ }^{42}$. Ainsi Hadès, secondé par Perséphone, aurait représenté à lui seul cette catégorie nébuleuse nommée par des Modernes «dieux chthoniens ». Cependant, il ne faut pas oublier un détail «significatif», comme dirait Marcel Detienne : car si Homère ne connaît pas de dieux Chthonioi, il parle, en revanche, d'un Zeus Katachthonios, d'un Zeus «dans la terre », « infernal », d'autant plus qu'il est mentionné avec la « redoutable Perséphone $»^{43}$. 
Il ne me semble pas inopportun de s'arrêter un instant sur ce Zeus Katachthonios, qu'on identifie souvent, à tort, avec Zeus Chthonios. Tout d'abord, on pourrait penser que Zeus, « père des dieux et des hommes » (en termes d'autorité), qui avait reçu, comme le rappelle Poséidon, «le vaste ciel, dans l'éther et les nuages $»^{44}$, ce dieu suprême donc est capable d'être partout, de couvrir tous les espaces: le ciel, le monde visible, le monde d'en bas. C'est ainsi que, d'une certaine façon, l'a compris Eustathe en commentant ce vers de l'Iliade, mais en identifiant aussi Zeus et Hadès :

Parce que le Zeus ici katachthonios est Hadès, comme si l'on disait air souterrain (aêr hupogeios). Selon un ancien récit, près d'un fleuve carien, il y avait un sanctuaire de Zeus Poséidon, et le mot Zeus, c'est-à-dire le mot Zên est un nom commun aux frères Zeus, Poséidon et Hadès ${ }^{45}$.

On voit qu'Eustathe ne confond pas katachthonios avec chthonios (mot qu'il n'emploie pas d'ailleurs), mais il identifie ce Zeus Katachthonios à Hadès, en considérant que le mot «Zeus », ou plus exactement Zńv (ou Zñv) ${ }^{46}$, constitue un nom commun aux trois frères. Par ailleurs, Eustathe identifie katachthonios (litt. «dans, au fond de la terre»), avec hupochthonios et hupogeios, "sous la terre ».

Deux textes de Pausanias nous aident à voir plus clair dans cette histoire d'un Zeus qui saurait être triple. Dans sa description de l'agora de Corinthe, le Périégète mentionne

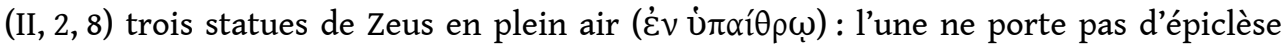
( plus haut $»)^{47}$. Or, dans son édition du livre II de Pausanias, George Roux - et il n'est pas le seul - traduit Chthonios par «Infernal », tandis que d'autres, comme Domenico Musti et Mario Torelli, dans l'édition italienne, transcrivent Chthonios, sans se compromettre ${ }^{48}$. Toutefois, on pourrait dire que le Zeus sans épiclèse représente peut-être ce dieu suprême, qui domine tout, qui se situe au-delà des particularités locales, je dirais un Zeus panhellénique, familier à tout Grec. L'épiclèse Hupsistos désigne certes Zeus comme le dieu du plus haut rang, mais elle rappelle aussi que Zeus est une divinité honorée souvent sur les sommets. Quant à l'épiclèse Chthonios, rien ne permet de l'entendre au sens de l'« Infernal », en l'identifiant au terme Katachthonios. Je rappelle que cette traduction, répétée souvent, vient du fait qu'on ne donne qu'un sens au mot chthonios. Cependant, comme nous l'avons déjà dit, cette épiclèse pourrait renvoyer à une association entre Zeus et la terre, la chthôn, en tant qu'humus fertile, en tant que sol qui produit de la richesse.

16 Le deuxième texte de Pausanias nous conduit, cette fois, à l'acropole d'Argos nommée Larissa (actuellement: Kastro), du nom d'une fille du roi Pelasgos (II, 24, 3-4). À son sommet, Pausanias mentionne un temple d'Athéna "qui est digne d'être vu» ( $\theta \dot{\varepsilon} \alpha \varsigma$

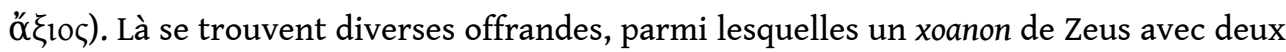
yeux dans leur position naturelle, et un troisième sur le front. On dit - continue Pausanias - que ce Zeus était un dieu patrôios de Priam, fils de Laomédon, et qu'il était

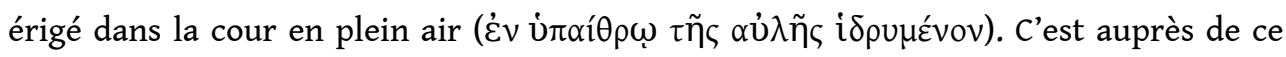
xoanon divin que se refugia Priam lorsqu'llion était en train de tomber dans les mains des Grecs, et c'est en tant que butin de guerre que cette image ancienne se trouvait là, sur la Larissa d'Argos. Or, à propos des trois yeux, le Périégète dit qu'on pourrait faire l'hypothèse suivante :

Que Zeus règne au ciel, cela est une tradition commune à tous les humains ( $\pi \alpha{ }^{\prime} v \tau \omega v$

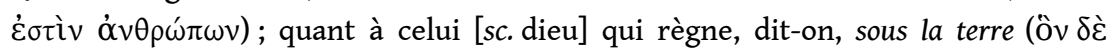

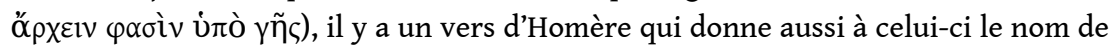
Zeus: Zeus souterrain et redoutable Perséphone ${ }^{49}$. Eschyle, le fils d'Euphoriôn, appelle 


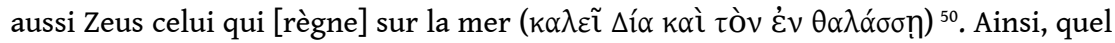
que soit celui qui a fait [le xoanon], pour qu'il voie avec trois yeux, il voulait signifier que le même dieu gouverne dans ce qu'on appelle les trois «lotissements » du monde ${ }^{51}$.

17 Remarquons tout d'abord que ce xoanon se trouve dans le temple d'Athéna. Le père est logé ainsi chez sa fille dont il devient sunnaos, bien que Pausanias signale aussi sur l'acropole un temple de Zeus Larissaios, mais qui, à l'époque du Périégète, est sans toit et sans la statue du dieu. Ensuite, ce Zeus avec trois yeux pourrait nous faire penser aussi à un autre pouvoir de ce dieu: Zeus, comme le Soleil, peut voir tout, son regard embrasse tout l'univers, c'est pour cela aussi qu'on invoque Zeus dans le serment, puisqu'il peut, entre autres, voir et punir partout le parjure. Cette capacité de tout voir est exprimée par une série d'épithètes de Zeus : il est qualifié par Eschyle de pantoptês ou panoptês ("qui voit tout»), il est celui dont le regard perce tout, celui qui observe d'en haut toute chose comme le dit Aristophane, ce que le scholiaste résume par le mot pantepoptês ${ }^{52}$. Or, c'est justement en ce sens qu'Hésychius a compris l'épithète panoptês (s.v.), qu'il explique par poluophthalmos, Zeus « qui a beaucoup d'yeux ».

Ce n'est cependant pas l'explication que donne Pausanias, qui pense plutôt à une triple nature du dieu. Plus tard, Proclus va, à sa façon, expliciter la même idée, en présentant Zeus comme le premier des Kronides, «créateur de toutes choses " (ǒ $\lambda \omega v$ $\omega v$

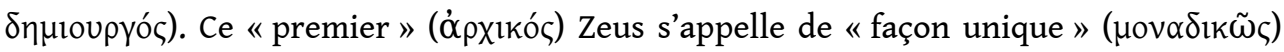

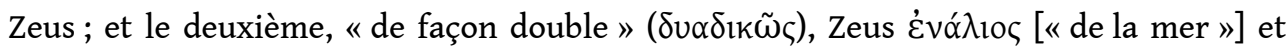
Poséidon; et le troisième, « de façon triple $(\tau \rho \imath \alpha \delta \kappa \tilde{\omega} \varsigma)$, Zeus katachthonios et Ploutôn et Hadès $»^{53}$.

Ces brèves remarques sur Zeus suggèrent, à mon sens, que lorsque les Grecs veulent parler d'un Zeus "du monde d'en bas ", d'un Zeus "souterrain ", qui règne hupo gês comme le dit Pausanias, ils ne font pas appel à l'épithète Chthonios: ils le qualifient de Katachthonios. Ajoutons que certains dieux, appelés parfois «chthoniens » par des Modernes - même si les textes ne mentionnent pas le mot chthonioi - sont, en fait, désignés par les Grecs de diverses façons, comme des dieux d'«en bas », ceux qui " commandent sous terre », ceux qui vivent dans une " demeure souterraine », etc. ${ }^{54}$.

\section{Olympe et les « Olympiens » homériques}

Venons maintenant rapidement à ce qu'on appelle les « dieux Olympiens » chez Homère. Car, chez les Modernes, cette expression revient de façon récurrente, presque spontanée, lorsqu'on veut parler en général des « dieux d'Homère ». Certes, on sait bien que, dans les poèmes homériques, les dieux sont associés, liés à l'olympe, et même étroitement. Il est plusieurs fois question de dieux qui « echousi Olumpon », c'est-à-dire qui «ont», qui « possèdent» l'Olympe ${ }^{55}$, ce qui signifierait aussi qu'il exercent leur pouvoir sur ce mont, " couvert de neiges abondantes » (aganniphos), qualifié de " haut » (makros), d'« immense » (megas), d'« éclatant» (aiglêeis), " aux nombreuses cimes » (poludeiras), " aux nombreux replis » (poluptuchos), etc. ${ }^{56}$. L'Olympe " escarpé » (aipus) constitue le "siège ", le hedos des immortels ; c'est là où ils " possèdent » leurs dômata olympiens, leurs «maisons", mais leurs assemblées, ils les tiennent souvent dans la "demeure» de Zeus, appelée domos, megara, dôma, dô ${ }^{57}$. C'est de l'olympe que descendent certaines divinités, comme Hermès, Iris ou Athéna, pour aller vers les mortels sur la terre, et c'est vers l'olympe que se dirigent certaines puissances divines, 
comme Thétis lorsqu'elle émerge du flot marin et monte "sur le grand ciel et l'Olympe " pour implorer Zeus et plaider, auprès de lui, la cause de son fils Achille ; ou comme la déesse Eôs (l'Aurore), quand elle s'avance «vers le haut Olympe, pour annoncer la lumière du jour à Zeus et aux autres immortels $\|^{58}$.

Cependant, il n'est pas inintéressant de remarquer que ces dieux qui possèdent et habitent l'Olympe, selon plusieurs passages, ne sont pas qualifiés d'«Olympiens", d'Olumpioi, sauf dans deux cas - si je ne me trompe pas : tout d'abord, au chant I de l'Iliade, il est question d'un temps éloigné où les "Olympiens », comme Héra, Poséidon ou Pallas Athéna, voulaient enchaîner Zeus, avant que Thétis ne vienne soustraire le dieu à ces chaines ${ }^{59}$. Et ensuite, au chant XX, lorsque les Olumpioi, incités par Zeus, vont rejoindre les combattants des deux armées, Achéens et Troyens, pour porter secours chacun à l'un des deux partis, selon sa préférence ${ }^{60}$. Cependant, si l'adjectif Olumpioi se fait si rare ${ }^{61}$, en revanche le terme Olumpios est mentionné plusieurs fois et toujours pour qualifier Zeus. C'est Zeus qui est le dieu Olympien par excellence, au point qu'il suffit souvent de cette seule épithète pour désigner Zeus. Par exemple, Nestor s'adresse à Olumpios pour qu'il écarte des Achéens "le jour implacable "; Héra appelle ainsi son époux lorsqu'elle lui demande de lui jurer un " puissant serment "; et Athéna demande à Olumpios, son père, pourquoi "son cœur ne se laisse pas émouvoir» devant les malheurs d'Ulysse ${ }^{62}$.

En tant qu'Olumpios, Zeus agit, choisit, décide, ordonne, en dominant tout : il «lance l'éclair " (asteropêtês); il " amène à la lumière » un "grand signe » (mega sêma), tel un serpent; il « fait nourrir en lui [sc. en Pâris] un grand malheur pour les Troyens, le magnanime Priam et ses enfants »; il choisit à qui des deux (Hector ou Achille) il va donner la « gloire » (euchos); il « commande », il « ordonne », de « délivrer » le corps du « divin Hector »; c'est lui qui « donne des souffrance » à Pénélope, mais c'est lui aussi qui « répartit le bonheur aux humains, aux bons comme aux mauvais, à chacun comme il veut $"^{63}$. Il est sans doute significatif qu'aucune déesse, chez Homère, ne soit gratifiée d'une telle épithète, excepté les Muses, "filles de Zeus qui tient l'égide ", qualifiées d'Olumpiades. Mais elles sont les seules, parmi les enfants du dieu, à être appelées ainsi ${ }^{64}$.

Cette omniprésence de Zeus Olumpios chez Homère, on la retrouve chez Hésiode. Certes, il y est souvent question de l'olympe, ou des immortels qui " possèdent » leurs dômata olympiens, ou même des " dieux venant de l'olympe " ${ }^{65}$. Cependant, là aussi, c'est Zeus qui semble avoir le droit exclusif d'être qualifié d'olumpios, un droit dont jouissent également ses filles, les Muses, comme chez Homère ${ }^{66}$. Or, ce Zeus Olumpios hésiodique est doublement intéressant, car il joue aussi un rôle particulier dans la réussite des travaux agricoles. En effet, comme nous l'avons déjà vu, Hésiode recommande à son frère Persès de prier Zeus Chthonios et l'hagnê Déméter lorsqu'« il commence le premier labour ", pour que "le fruit sacré de Déméter soit mûr et alourdi $»^{67}$. Il apparaît cependant que ces deux divinités ne suffisent pas pour une bonne récolte. Car, après

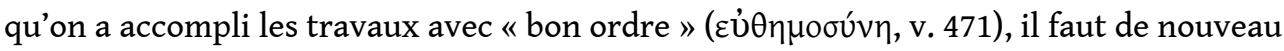
l'aide de Zeus, mais cette fois de Zeus Olumpios : s'il consent à donner par la suite un bon résultat, les épis se pencheront avec vigueur vers la terre ${ }^{68}$.

Un examen plus approfondi pourrait creuser sans doute mieux les raisons de cette relation privilégiée entre Zeus et le qualificatif Olumpios. Tout au moins, on pourrait dire qu'en accaparant cette épithète, Zeus impose son pouvoir aux autres dieux : il apparaît comme le possesseur incontesté et le plus important de l'olympe, comme ce 
dieu suprême qui rassemble d'autorité les autres dieux dans son domos, dans sa «demeure » olympienne pour leur parler et imposer ses volontés. Or, la connivence entre Zeus et cette dénomination serait perceptible aussi sur le plan cultuel, après Homère. Certes, un survol rapide de cette question pourrait montrer que l'épiclèse Olumpia peut qualifier aussi certaines déesses, comme Aphrodite, Artémis, Déméter, Eileithyia, Nikè, et surtout Gê ou Héra, mais ces cas sont limités et, dans leur grande majorité, tardifs ${ }^{69}$. Toutefois, c'est toujours Zeus qui, de façon écrasante, domine en tant qu'Olumpios, et non seulement à Olympie ${ }^{70}$ ou à Athènes, mais aussi dans plusieurs autres cités, en Grèce propre, en Asie mineure, en Italie ou ailleurs. Quant à l'expression générale «les dieux olympiens », elle n'est pas, certes, absente des textes et des inscriptions ${ }^{71}$; mais l'examen de cet ensemble divin, après Homère, ne s'inscrit pas dans le cadre de cet article.

\section{Les dieux et le « large » ciel}

25 À la fin de cet excursus homérique, semé parfois de quelques digressions, j'aimerais tout juste noter un autre aspect qui aurait nécessité une recherche à part. On constate, en effet, qu'à ce schéma géographique, qui va de l'olympe au monde d'en bas en passant par la terre, vient se mêler un autre espace, à savoir le ciel, ouranos. Qualifié de « vaste » (megas), d'« étoilé » (asteroeis), mais surtout de «large » (eurus), il est parfois décrit comme un ciel «d'airain » (chalkeos) vers lequel s'élève, pendant la bataille, le

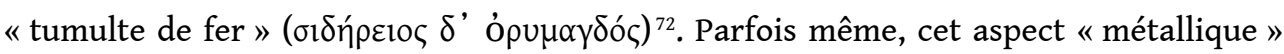
du ciel est davantage prononcé : l'ouranos est qualifié alors de poluchalkos, "plein, couvert de beaucoup d'airain », ou encore "de fer» (sidêreos). Or, c'est vers ce ciel "dur ", pourrait-on dire, et menaçant que monte la poussière que soulèvent les pieds des chevaux en frappant le sol, mais c'est aussi jusqu'à cet ouranos que parviennent l'hubris et la violence des prétendants, dans l'Odyssée, ce qui suggère l'énormité, l'étendue de leur comportement outrageant ${ }^{73}$. Cependant, ce ne sont pas seulement le

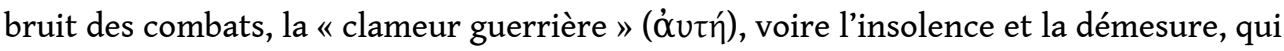
arrivent jusqu'au ciel. C'est aussi la "gloire», le kleos, qui monte à l'ouranos : non seulement le kleos de la geste humaine que chante l'aède, ou la " gloire » d'un valeureux comme Ulysse, ou encore d'une femme "très sage » ( $\pi \varepsilon \rho i ́ \varphi \rho \omega v)$ telle Pénélope, mais aussi le kleos d'un bouclier « tout en or ", comme celui de Nestor ${ }^{74}$.

Comme pour l'olympe, le ciel et tout particulièrement le "ciel large " - selon la formule habituelle - est considéré aussi comme un domaine que les dieux immortels «ont", «possèdent" (echousi) ${ }^{75}$. C'est pourquoi ils sont parfois qualifiés de theoi Ouraniônes, ou epouranioi, "dieux du ciel», «dieux célestes $»^{76}$. Cependant, cette présence d'ouranos pose certaines questions qu'on ne saurait aborder ici, comme celle des rapports entre l'olympe et le ciel dont les portes étaient gardées par les Heures, car c'est à elles, aux Horai, qu'on avait confié " le grand ciel et l'Olympe ${ }^{77}$ »; ou encore celle de la relation entre ces deux espaces, céleste et olympien, d'une part et, de l'autre, ce qu'on appelle éther, présenté aussi comme siège de Zeus Olumpios $^{78}$. Plus précisément, se pose également le problème des relations entre les dieux et le ciel, qui sont parfois semblables aux rapports entre ces immortels et l'olympe : à titre d'exemple, les dieux, tels Athéna et d'autres, peuvent « descendre » du ciel, ouranothen, ex ouranou, comme ils descendent de l'olympe, pour apporter parfois leur aide aux Troyens ou aux Achéens ${ }^{79}$. À noter cependant que c'est vers le ciel, et non pas vers l'olympe, que monte la knisê, la 
fumée et l'odeur de la viande sacrificielle brûlée sur l'autel pour les dieux ${ }^{80}$, et c'est vers le ciel que les hommes lèvent les yeux ou les mains, en adressant leurs prières aux dieux $^{81}$.

Il est sans doute significatif que ce soit surtout à Zeus qu'on adresse des prières lorsqu'on lève les yeux et les mains vers le ciel. Car, en fait, c'est encore Zeus le maître absolu du ciel. Dans le fameux partage de l'univers entre les trois frères, c'est Zeus qui a obtenu, après tirage au sort, « le large ciel, en plein éther et nuages », tandis que la mer revient à Poséidon et les régions ténébreuses et humides du monde d'en bas à Hadès ${ }^{82}$. Cependant, comme le précise Poséidon, irrité contre Zeus qui veut lui imposer ses ordres, la terre (gaia) et le haut Olympe constituent des biens communs à tous les trois frères. Ils peuvent donc circuler librement sur la terre, monter sur l'olympe et descendre de cette haute montagne, sans être sous la tutelle de Zeus, ce que dit en substance Poséidon. Ainsi, on voit même Hadès aller sur l'olympe pour être guéri par Péon, le médecin des dieux, après avoir été frappé d'une flèche par Héraclès à Pylos ${ }^{83}$. Il n'empêche que c'est Zeus qui est reconnu comme le "suprême dirigeant " (hupatos mêstôr) qui, «du haut du ciel» (ap'ouranothen), agit et prononce ses sentences, étend pour les mortels un arc-en-ciel empourpré (porphureê iris), ou « fait retentir son terrible tonnerre ", quand il ne décide pas de le faire éclater du haut de l'olympe ${ }^{84}$.

C'est ainsi que les divinités homériques parcourent une vaste étendue qui va en particulier du ciel à la terre en passant par l'olympe, leur demeure principale. Polyvalents, accomplissant diverses fonctions, choisissant leurs alliés ou leurs ennemis, les dieux d'Homère vont et viennent surtout entre leurs dômata olympiens et la gaia des humains. Il est bien clair, je l'espère, que ce texte, humble hommage à notre ami et collègue disparu, n'a pas la prétention de présenter une étude substantielle sur Les dieux d'Homère ${ }^{85}$. À partir d'un examen préliminaire d'une série de termes homériques, j'ai voulu seulement entamer quelques réflexions, en m'interrogeant, entre autres, sur cette tendance, pas si rare, de vouloir « caser » les divinités grecques dans des catégories parfois étanches, qui s'accordent mal avec la "polyphonie» religieuse des Grecs.

\section{BIBLIOGRAPHIE}

BALLABRIGA Alain, Le soleil et le Tartare : l'image mythique du monde en Grèce archaïque, Paris, EHESS, 1986.

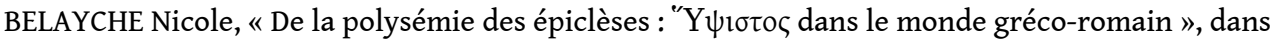
N. Belayche, P. Brulé, G. Freyburger, Y. Lehmann, L. Pernot \& F. Prost (éd.), Nommer les dieux. Théonymes, épithètes, épiclèses dans l'Antiquité, Rennes / Turnhout, Presses universitaires de Rennes / Brepols, 2005, p. 427-442.

BURKERT Walter, Griechische Religion der archaischen und klassischen Epoche, Stuttgart, Kohlhammer, 2011 ${ }^{2}$ (1 $1^{\text {re }}$ éd. 1977). 
CGRN = J.-M. CARBON, S. PEELS \& V. PIRENNE-DELFORGE (éd.), Collection of Greek Ritual Norms, Liège, 2016- (<http://cgrn.ulg.ac.be>).

DELG = CHANTRAINE Pierre, Dictionnaire étymologique de la langue grecque, Paris, Klincksieck, 1968-1980.

FAIRBANKS Arthur, « The Chthonic Gods of Greek Religion », AJP, 21, 1900 (3), p. 141-159.

FENET Annick, Les dieux olympiens et la mer : espaces et pratiques culturelles, Rome, École française de Rome, 2016.

GEORGOUDI Stella, « Bachofen, le matriarcat et le monde antique. Réflexions sur la création d'un mythe ", dans G. Duby \& M. Perrot (éd.), Histoire des femmes en Occident, vol. I : L'Antiquité (sous la direction de P. Schmitt-Pantel), Paris, Plon, 1991, p. 477-491 et 555-557 (notes).

GEORGOUDI Stella, «Les Douze Dieux des Grecs : variations sur un thème », dans S. Georgoudi et J.-P. Vernant (éd.), Mythes grecs au figuré : de l'antiquité au baroque, Paris, Gallimard, 1996, p. 43-82. GEORGOUDI Stella, « Gaia/Gê. Entre mythe, culte et idéologie », dans S. des Bouvrie (éd.), Myth and Symbol I. Symbolic Phenomena in Ancient Greek Culture, Bergen, Norwegian Institute at Athens, 2002, p. 113-134.

GEORGOUDI Stella, «Chruseos kosmos. L'usage ambivalent de l'or dans les pratiques rituelles grecques ", dans M. Tortorelli Ghidini (éd.), AURUM. Funzioni e simbologie dell'oro nelle culture del Mediterraneo antico (Actes du colloque international, Naples, juin 2011), Rome, « L'Erma » di Bretschneider, 2014, p. 249-275.

GUTHRIE William K. C., The Greeks and Their Gods, Londres, Methuen, 1950.

HÄGG Robin \& ALROTH Brita (éd.), Greek Sacrificial Ritual, Olympian and Chthonian (Proceedings of the Sixth International Seminar on Ancient Greek Cult, organized by the Department of Classical Archaeology and Ancient History, Göteborg University, 25-27 April 1997), ActaAth-8 ${ }^{\circ}, 18$, Stockholm, 2005.

HARRISON Jane, Prolegomena to the Study of Greek Religion, $1922^{3}$ (1 $1^{\text {re }}$ éd. 1903, Cambridge University Press), réimpr. New York, Meridian Books, 1975.

LE GUEN-POLLET Brigitte, La vie religieuse dans le monde grec $d u v^{e}$ au III siècle avant notre ère. Choix de documents épigraphiques traduits et commentés, Toulouse, Presses universitaires de Mirail, 1991.

MIKALSON Ion D., Ancient Greek Religion, Oxford, Blackwell, 2005.

MUSTI Domenico \& TORELLI Mario (éd.), Guida della Grecia. Libro II: La Corinzia e l'Argolide, Milan, Mondadori, 1986.

NAGY Gregory, The Best of the Achaeans: Concepts of the Hero in Archaic Greek Poetry, Baltimore / Londres, The Johns Hopkins University Press, 1979.

PARKER Robert, On Greek Religion, Ithaka / Londres, Cornell University Press, 2011.

PIRENNE-DELFORGE Vinciane, L'Aphrodite grecque : contribution à l'étude de ses cultes et de sa personnalité dans le panthéon archaïque et classique, Athènes / Liège, Presses universitaires de Liège (Kernos, suppl. 4), 1994.

PIRENNE-DELFORGE Vinciane, Retour à la source. Pausanias et la religion grecque, Liège, Presses universitaires de Liège (Kernos, suppl. 20), 2008.

PIRENNE-DELFORGE Vinciane \& PIRONTI Gabriella, L'Héra de Zeus. Ennemie intime, épouse définitive, Paris, Les Belles Lettres, 2016. 
PIRONTI Gabriella, « De l'éros au récit : Zeus et son épouse », dans G. Pironti \& C. Bonnet (éd.), Les dieux d'Homère. Polythéisme et poésie en Grèce ancienne, Liège, Presses universitaires de Liège (Kernos, suppl. 31), 2017, p. 63-83.

PIRONTI Gabriella \& BONNET Corinne (éd.), Les dieux d'Homère. Polythéisme et poésie en Grèce ancienne, Liège, Presses universitaires de Liège (Kernos, suppl. 31), 2017.

ROBERTSON Noel, « Sacrifice to the Sea: A Custom Prior to the "Olympian" and "Chthonian" Categories », dans R. Hägg \& B. Alroth (éd.), Greek Sacrificial Ritual, Olympian and Chthonian (Proceedings of the Sixth International Seminar on Ancient Greek Cult, organized by the Department of Classical Archaeology and Ancient History, Göteborg University, 25-27 April 1997), ActaAth-8 ${ }^{\circ}, 18$, Stockholm, 2005, p. 85-98.

ROHDE Erwin, Psyche. The Cult of Souls \& Belief in Immortality among the Greeks, trad. angl., 2 vol., New York, Harper \& Row, 1966 ( $1^{\text {re }}$ éd. 1925, Londres / New York).

ROUX Georges, Pausanias en Corinthie : Livre II, 1 à 15, Paris, Les Belles Lettres, 1958.

RUTHERFORD Ian, «Canonizing the Pantheon: The Dodecatheon in Greek Religion and Its Origins ", dans I. N. Bremmer \& A. Erskine (éd.), The Gods of Ancient Greece. Identities and Transformations, Édimbourg, Edinburgh University Press, 2010, p. 43-54.

SCHLESIER Renate, « Prolegomena to Jane Harrison's Interpretation of Ancient Greek Religion », dans W. M. Calder (éd.), The Cambridge Ritualists Reconsidered, Atlanta, Scholars Press, 1991, p. 185-226.

SCHLESIER Renate, « Olympian versus Chthonian Religion », SCI, 21, 1991-1992, p. 38-51.

SCHLESIER Renate, « Olympische Religion und chthonische Religion », dans U. Bianchi (éd.), The Notion of "Religion" in Comparative Research (Selected Proceedings of the XVI IAHR Congress, Rome, 3-8 septembre 1990), Rome, « L’Erma » di Bretschneider, 1994, p. 301-310.

SCULLION Scott, « Olympian and Chthonian », ClAnt, 13, 1994 (1), p. 75-119.

SCULLION Scott, « "Saviours of the Father's Hearth": Olympian and Chthonian in the Oresteia », dans R. Hägg \& B. Alroth (éd.), Greek Sacrificial Ritual, Olympian and Chthonian (Proceedings of the Sixth International Seminar on Ancient Greek Cult, organized by the Department of Classical Archaeology and Ancient History, Göteborg University, 25-27 April 1997), ActaAth-8 ${ }^{\circ} 18$, Stockholm, 2005, p. 23-36.

SOKOLOWSKI Franciszek, Lois sacrées des cités grecques (LSCG), Paris, École française d'Athènes, 1969.

WEST Martin L. (éd.), Hesiod, Works and Days, Oxford, Clarendon Press, 1978.

ZANETTO Giuseppe \& Dario DEL CORNO (éd.), Aristofane. Gli Uccelli, Milan, Mondadori, 1987.

\section{NOTES}

1. À vrai dire, ce n'est pas moi qui invente ces qualificatifs ! Sophocle l'avait bien formulé dans

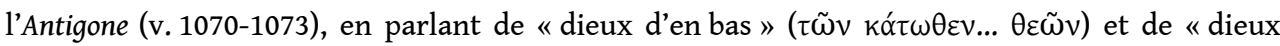

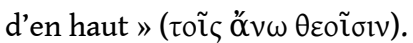

2. Guthrie $(1950,209)$ - je souligne en caractères droits.

3. Schlesier (1991-1992 et 1994).

4. Sur Bachofen et la théorie du matriarcat, cf. Georgoudi (1991). 
5. Harrison (1922), en particulier : chapitre I, 1-31 («Olympian and Chthonic Ritual»), et chapitre VI, 257-321 («The Making of a Goddess»). Sur ce schéma structural et, plus généralement, sur des travaux des savants qu'elle discute, voir Schlesier (1991, 210-218, avec références bibliographiques).

6. Rohde (1966, I, 25, 158-161; II, 452, n. 75).

7. Cf., par exemple, Parker (2011, 80-84).

8. Cf. Scullion $(2005,24)$, qui parle de " "mingling" of Olympian and chthonian », ce qui rappelle le Doppelwesen (Olympien / Chthonien) de Burkert (2011, 319), cité aussi par Scullion (2005, 25-26). Cf. aussi Scullion (1994, 90-92).

9. Voir Mikalson (2005, 38-39). D'autres continuent à parler d'« "Olympian" and "Chthonian" Categories", ou d'«Olympian» et "chthonian» sacrifice, en se contentant de mettre ces qualificatifs entre guillemets, sans autre commentaire : cf. Robertson $(2005,85,90)$.

10. J'ajoute, entre parenthèse, que ces clichés ont la vie dure, car ils fleurissent et se répandent au-delà de l'univers des spécialistes. En effet, il suffit de taper le mot chthonien sur Google, pour trouver la définition suivante, rédigée en août 2016 dans la Wikipédia : "Les divinités grecques chthoniennes ou telluriques sont des divinités anciennes ayant contribué à la formation du Panthéon grec. Elles sont dites "chthoniennes" (du grec ancien $\chi \theta \omega ́$ v / khthôn, "la terre") ou “telluriques" (du latin tellus, « la terre ») parce qu'elles se réfèrent à la terre, au monde souterrain ou aux Enfers, par opposition aux divinités célestes, dites "ouraniennes" ou "éoliennes" [de Éole, dieu du vent] $»-$ je souligne.

11. Scullion (2005, 23-24) - je souligne en caractères droits.

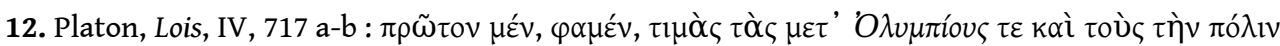

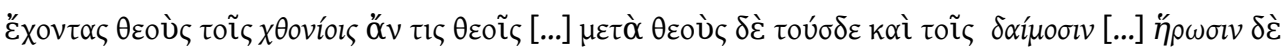

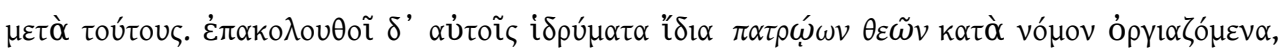

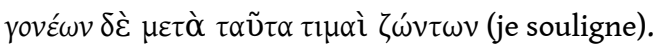

13. Cf., parmi d'autres exemples, Scullion (1994, 76 : les « chthonian powers" sont «the gods of the underworld, heroes, and the dead»). Qui plus est, en discutant les positions de Fairbanks (1900), Scullion $(1994,93)$ ajoute aussi, à ces " puissances chthoniennes ", " the agricultural gods », créant ainsi une sorte d'indistinction entre les différentes parties de la terre (voir infra).

14. Sur les similitudes entre chthôn et gaia/gê, autre nom de la "terre », cf. Georgoudi (2002, surtout p. 117, ainsi que la n. 14).

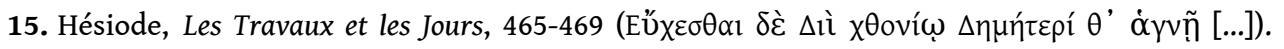
Pourtant, le Zeus Chthonios de ce passage est considéré comme un Zeus «Infernal ", selon la traduction de Mazon (CUF), mais aussi selon West, dans son commentaire des Works and Days $(1978,276)$, où il semble confondre le Chthonios avec le Katachthonios. Cf. aussi le commentaire d'Anne Jacquemin, dans son édition du livre $V$ de Pausanias (CUF), où il est question d'un autel de Zeus Chthonios $(\mathrm{V}, 14,8)$ : «Zeus Chthonios, dieu infernal, est associé par Hésiode $(T J, 465)$ dans la prière qui prélude aux labours, alors que Homère connaît un Zeus Catachthonios » (p. 184, je souligne).

16. Sokolowski, LSCG, $\mathrm{n}^{\circ} 96$ / CGRN 156, 1. 24-25 (ca 230-200 av. notre ère).

17. Scullion $(1994,88)$ reconnaît « an agricultural connection» par rapport à ce Zeus Chthonios (mais voir supra, n. 13, sur « the agricultural gods»).

18. Classer ainsi Zeus Chthonios et Gê Chthoniê dans la soi-disant "catégorie des dieux chthoniens ", même si l'on ne se trompait pas, en l'occurrence, sur le sens de ces épiclèses, peut créer, me semble-t-il, une confusion inutile.

19. Cf. Le Guen-Pollet (1991, 192, n. 13 : « la couleur noire est souvent attestée pour les victimes

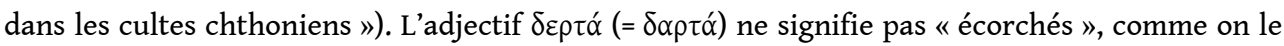
traduit parfois, mais ceux qu'on peut écorcher.

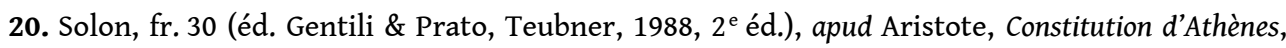

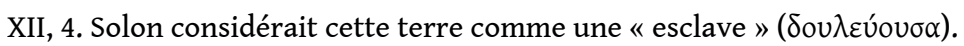


21. Dans son commentaire du calendrier de Myconos (CGRN 156), Jan-Mathieu Carbon met justement en relation la couleur de cette terre avec la peau noire des animaux sacrifiés à Zeus Chthonios et à Gê Chthoniê.

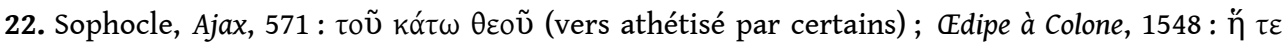

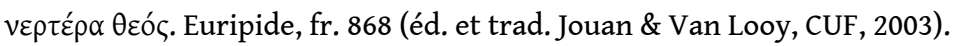

23. Sur le Tartare, cf., entre autres, Ballabriga (1986).

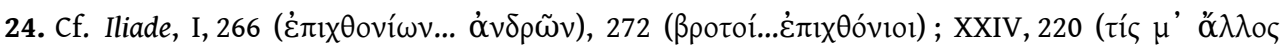

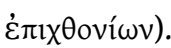

25. Cf., par exemple, eureia : Iliade, XXI, 387 ; euruodeiê: Iliade, XVI, 635 ; Odyssée, III, 453 ; X, 149 ; XI, 52 ; phul' anthrôpôn : Odyssée, VII, 307.

26. Sur pouluboteira, cf. Iliade, III, 195, 265 ; VI, 213 ; XI, 619 ; XII, 158, etc. ; Odyssée, VIII, 378 ;

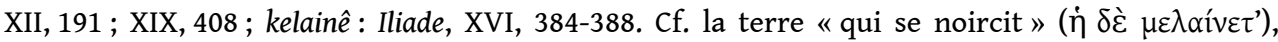

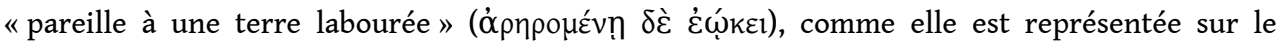
fameux bouclier d'Achille forgé par Héphaïstos (Iliade, XVIII, 548-549). Mais le dieu façonne cette

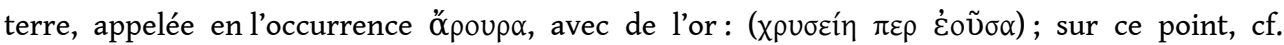

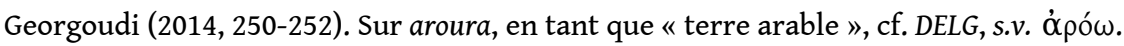

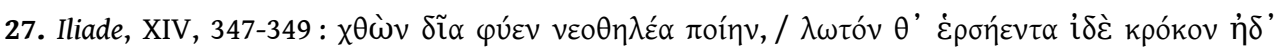

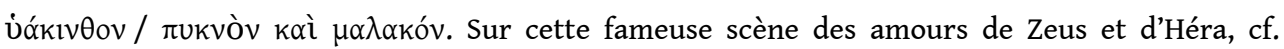
Pirenne-Delforge \& Pironti (2016, 42-46, 237-243) et Pironti (2017).

28. Cf. brotoi : Odyssée, VI, 153 ; VIII, 222 ; tomber sur la terre : Iliade, XII, 190-194 ; XIV, 438-439; XX, 482-483, etc. ; Odyssée, XXII, 296 ; serment d'Héra : Iliade, XIV, 270-272.

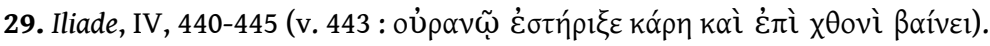

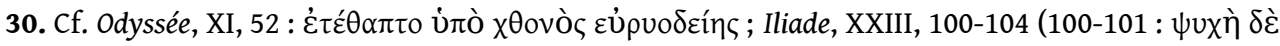

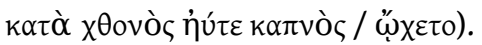

31. Cf. poluphorbos : Iliade, IX, 568 ; XIV, 200, 301 ; apeiresiê : Iliade, XX, 58 ; apeirôn : Iliade, VII, 446 ;

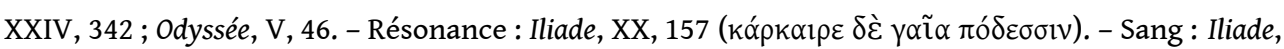

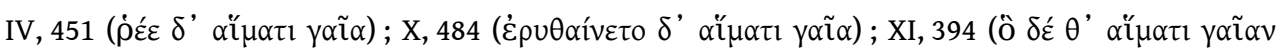
$\dot{\varepsilon} \rho \varepsilon v ́ \theta \omega v)$, etc. Cependant, ce sang qui coule et «imprègne » la gaia est qualifié, à l'occasion, de

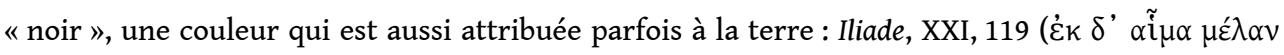

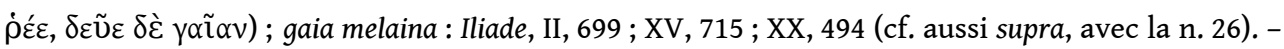

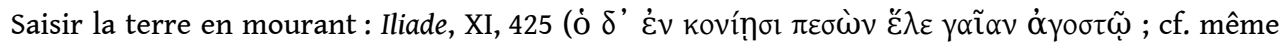
phrase : Iliade, XIII, 508, 520 ; XIV, 452).

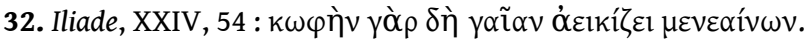

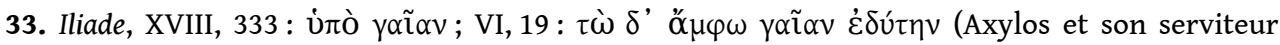

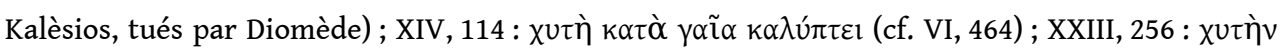

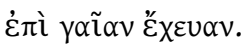

34. Comme il est qualifié dans l'Iliade, VIII, 208.

35. Iliade, XX, 57-65.

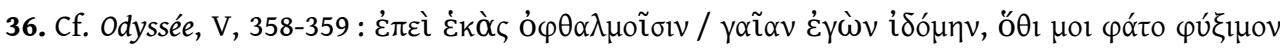
$\tilde{\varepsilon} \tilde{i}_{v a l}$ ( avec mes yeux j'ai vu au loin la terre, lieu de refuge pour moi, disait-elle [sc.

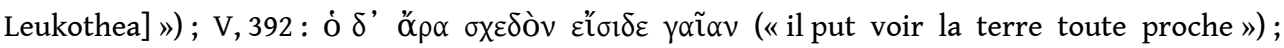

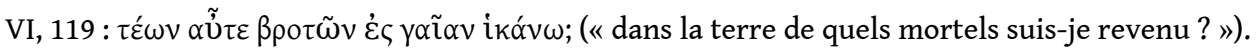

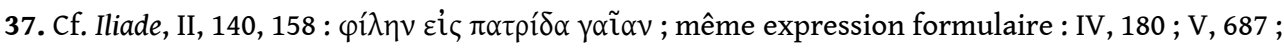

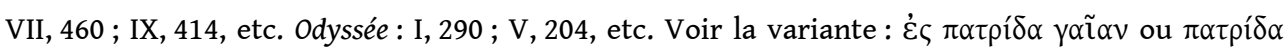
үã̃ $\alpha v$ (cf. Odyssée : III, 117 ; IV, 476, 545, 558, etc. ; V, 144, 207 ; VII, 77, 193 ; IX, 79 ; X, 33, etc.).

38. Sur cette question et sur la figure d'une déesse hypothétique, la soi-disant "Terre Mère ", promue par plusieurs historiens des religions au rang de divinité universelle des origines à laquelle tous les peuples auraient rendu un culte, et qui serait source unique de vie et d'énergie pour les hommes, les animaux et les végétaux, cf. Georgoudi (2002). 


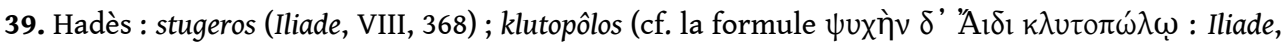

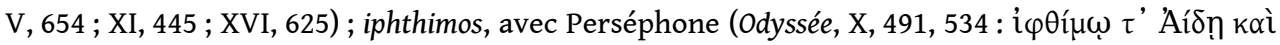

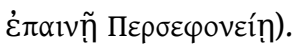

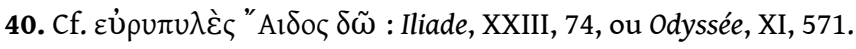

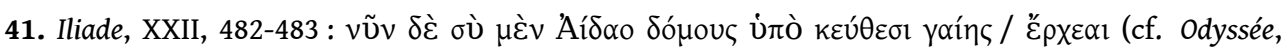

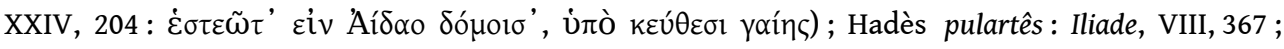

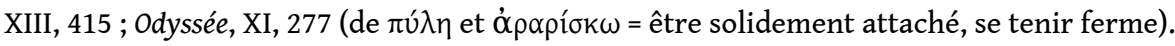

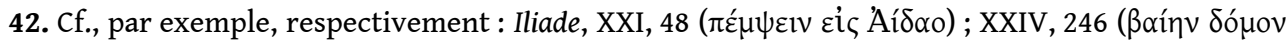

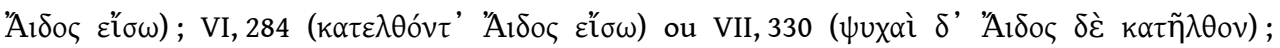

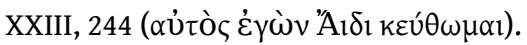

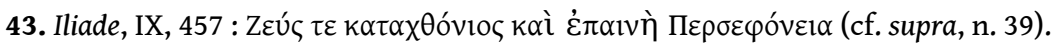

44. Iliade, XV, 192 (voir infra, avec la n. 82).

45. Eustathe, Commentarii ad Homeri Iliadem, IX, 457, p. 763, 50 ([...] ท̆

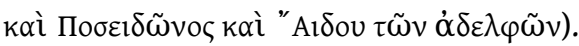

46. Autre forme du nom Zeus : cf. Eschyle, Suppliantes, 162.

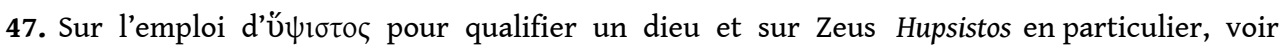
Belayche (2005).

48. Roux (1958); Musti \& Torelli (1986).

49. Sur ce vers d'Homère, voir supra, avec la n. 43.

50. Eschyle, fr. 464 Mette.

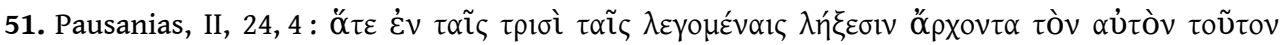
$\theta \varepsilon$ cóv. Le terme $\lambda \tilde{\eta} \xi ı$ signifie littéralement « la part assignée par le lot », une allusion peut-être à la répartition du monde entre les trois frères, Zeus, Poséidon, Hadès (cf. Iliade, XV, 184-199, où l'on trouve le verbe $\lambda \alpha \gamma \chi \alpha ́ v \omega$, «obtenir par le sort »). Sur ces passages de Pausanias, cf. aussi Pirenne-Delforge $(2008,260)$.

52. Eschyle, Suppliantes, 139 ( $\pi \alpha v \tau o ́ \pi \tau \alpha \varsigma)$; Euménides, 1045 ( $\pi \alpha v o ́ \pi \tau \alpha \varsigma)$. Aristophane, Acharniens,

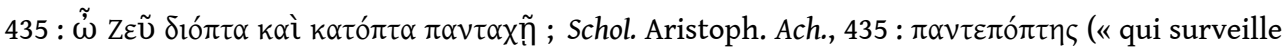
tout »).

53. Proclus, Platonis Cratylum Commentaria, CXLVIII (402 be-d), Teubner.

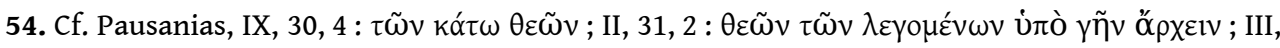

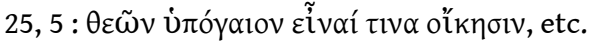

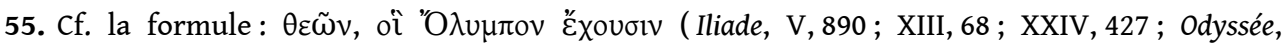

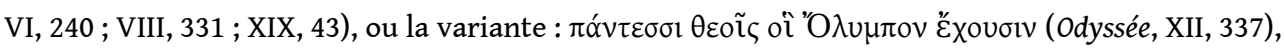

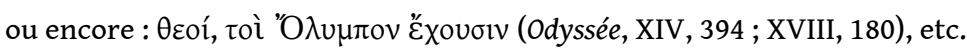

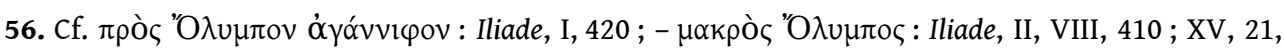

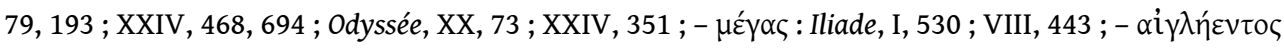

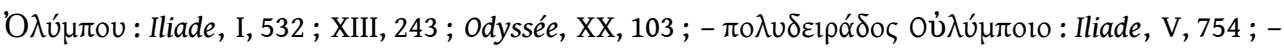

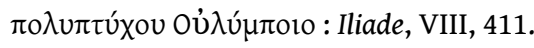

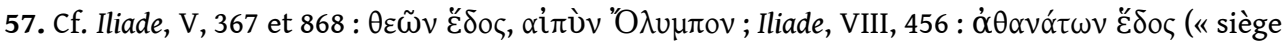

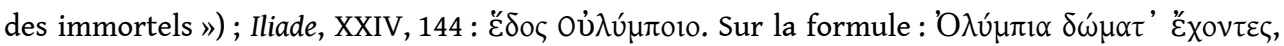
cf. Iliade, I, 18 ; II, 13 ; V, 384 ; XV, 115 ; Odyssée, XX, 79 ; XXIII, 167, etc. Sur la « demeure » de Zeus, où se rassemblent "tous ensemble" (athrooi) les dieux immortels, voir Iliade, XV, 84-85:

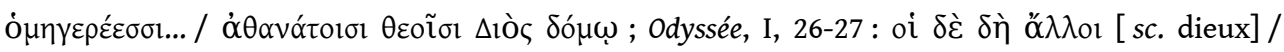

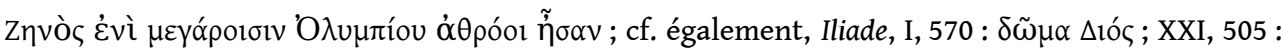
$\Delta$ òç... $\chi \alpha \lambda_{\kappa \circ} \beta \alpha \tau \dot{\varepsilon} \varsigma \delta \tilde{\omega}$ (la maison, le palais de Zeus, " au seuil d'airain »). Zeus convoque parfois

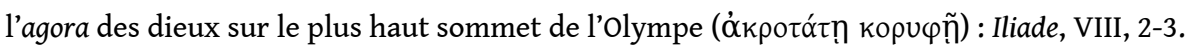

58. Cf., par exemple, Hermès (envoyé par Zeus à Troie) : Iliade, XXVI, 332-346. Athéna ou Iris (qui viennent de l'Olympe en courant, en tant que messagères) : Iliade, XI, 714-715 (Ä

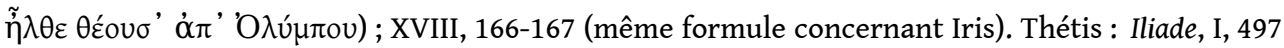




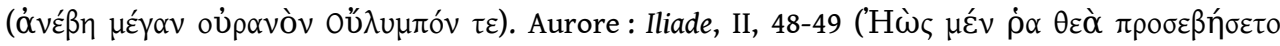

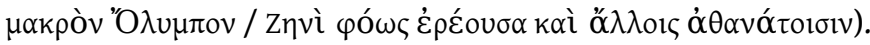

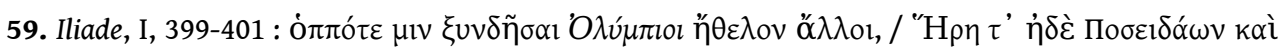

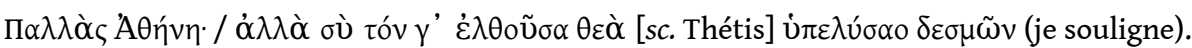

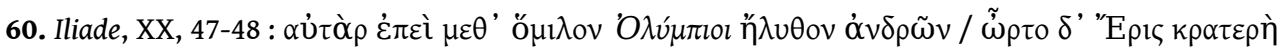
$\lambda \alpha$ $\alpha \sigma \sigma o ́$ ( («mais lorsque les Olympiens sont venus au milieu de la troupe des combattants, se leva la vigoureuse Éris, qui pousse les guerriers au combat ») (je souligne).

61. Ce qui n'empêche pas qu'on ait parlé de "dieux olympiens", quoiqu'en donnant à ce syntagme un sens extensif, comme le fait, par exemple, Nagy $(1979,7):$ : [...] the Homeric concept of the Olympian gods which incorporates, yet goes beyond, the localized religious traditions of each citystate. »Cf. aussi, plus récemment, Fenet $(2016,9-10)$ qui, par «divinités olympiennes », entend «les dieux censés appartenir au panthéon de l’olympe, désignés parfois sous l'expression de Douze Dieux ", une affirmation infondée ; sur ce point et sur la critique de la soi-disant «liste canonique » des Douze Dieux, cf. Georgoudi (1996). J'ajouterai que même si l'on accepte que les membres de cette liste varient, continuer à parler de l'«Olympian Dodekatheon » porte à confusion : voir, par exemple, Rutherford (2010, 46-47).

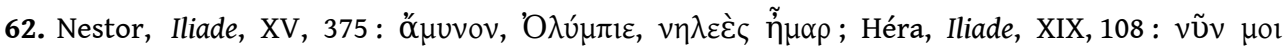

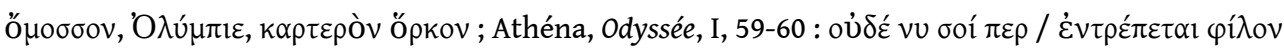

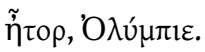

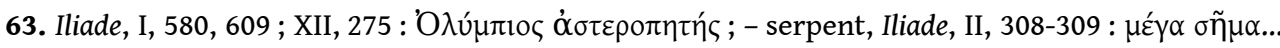

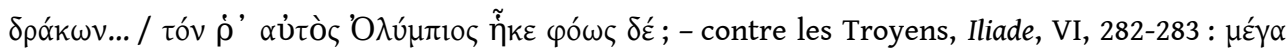

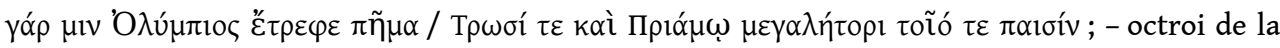

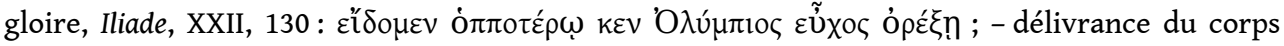

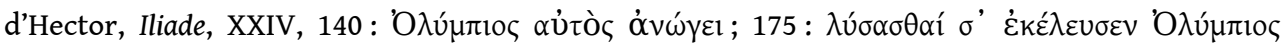

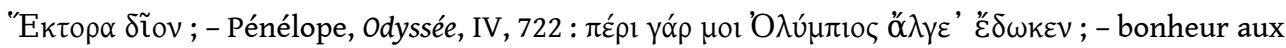

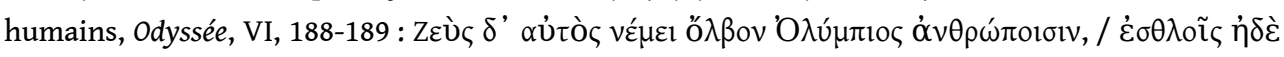

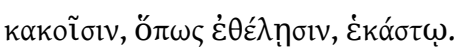

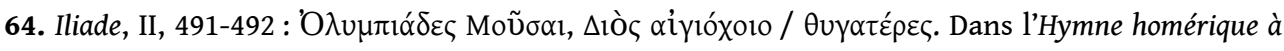

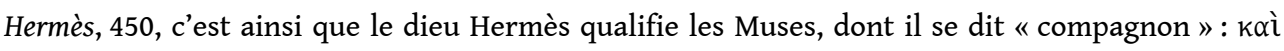

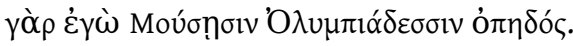

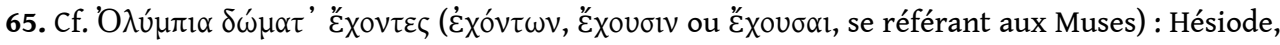
Théogonie, 75, 114, 783, 804, 963 ; Les Travaux et les Jours, 81, 110, 128, etc. Théogonie, 633 : oî $\delta$ ' ó $\rho$ '

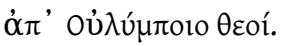

66. Zeus Olumpios : Hésiode, Théogonie, 529, 884 ; Les Travaux et les Jours, 87, 245 ; ou seulement Olumpios : Théogonie, 390 ; Les Travaux et les Jours, 474. Muses : Théogonie, 25, 52, 966, 1022 : Moṽ $\sigma 1$

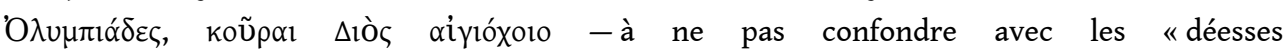

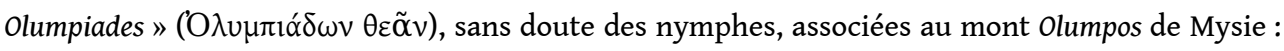
Sophocle, Ajax, 881. Cependant, d'après un fragment d'Hésiode (fr. 252, 2 West, apud Pausanias, IX, 40, 5-6 ; cf. aussi fr. 129,5), il semble que le poète qualifie, généralement, d'olumpiades les déesses habitant l'olympe : une fille d'Iolaos serait semblable, quant à son aspect, à ces divinités

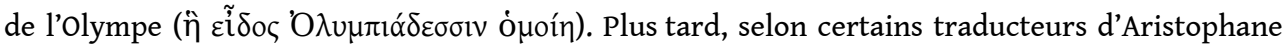
(Oiseaux, 781-783), cette épithète rare ('O $\lambda u \mu \pi ı \alpha ́ \delta \varepsilon \varsigma)$ va être attribuée aussi aux Charites, à côté des Muses ; cf. Zanetto \& Del Corno (1987), vers 781-782 : « il canto delle Grazie celesti ».

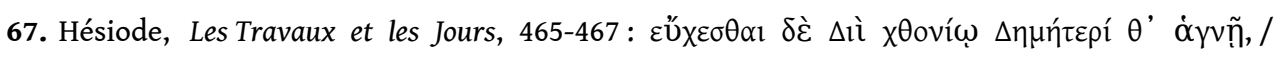

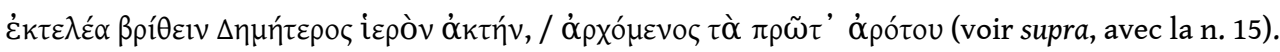

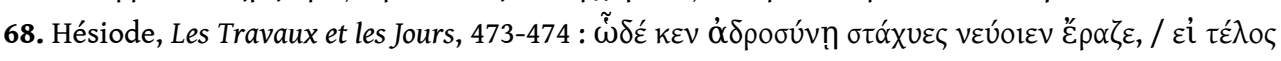

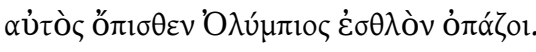

69. Aphrodite: à Sparte (statues de Zeus et d'Aphrodite, honorés sous l'épiclèse Olumpioi : Pausanias, III, 12, 11), ou à Tégée (Aphrodite Olumpia : IG V 2, nº 69); Artémis : à Érétrie (IG XII 9 , 


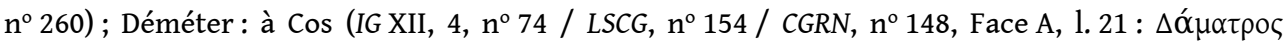

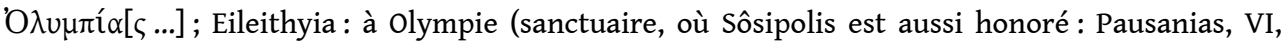

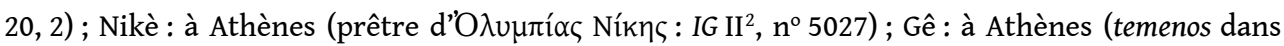
l'enceinte du sanctuaire de Zeus Olumpios: Pausanias, I, 18, 7, et sa prêtresse : IG II ${ }^{2}, \mathrm{n}^{\circ} 4521 \mathrm{a}$, 1. 10 : i $\varepsilon \rho \varepsilon i ́ \alpha \varsigma$ Г Г̃ $\varsigma$ 'O $\lambda u v \pi i ́ \alpha[\varsigma]$, etc.) ; Héra : à Olympie (autel de cendres : Pausanias, V, 14, 8), en

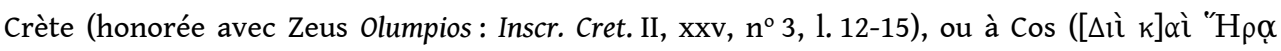

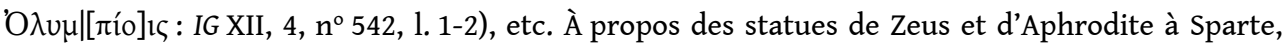
qui se trouvent près du lieu d'assemblée des citoyens, voir Pirenne-Delforge (1994, 196-197) qui pense, avec raison, que ces deux divinités peuvent avoir, en leur qualité d'olympiennes, «des prérogatives politiques $»$.

70. Cf. déjà au vi ${ }^{\mathrm{e}}$ siècle avant notre ère : CGRN, $\mathrm{n}^{\circ} 4$ (525-500), 1. 2-4 : $\Delta \mathrm{ì} \mathrm{O} \lambda u v[\pi i ́ o 1 . .$.$] .$

71. Cf., à titre d'exemple, Aristophane, Thesmophories, 331-332, 960-961 (adresser des prières aux

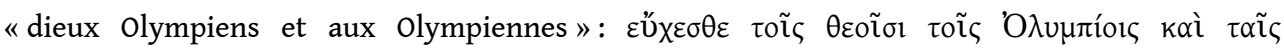

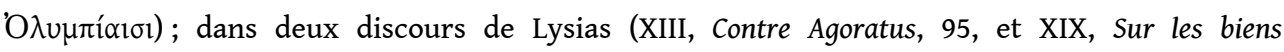

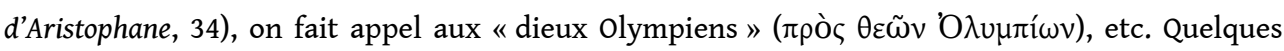
inscriptions, plutôt tardives (par exemple à : Ioulis de Kéos, Égine, Olympie, Chersonèse Taurique,

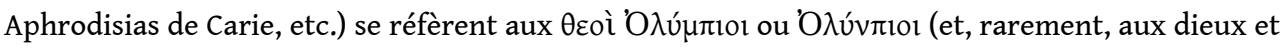

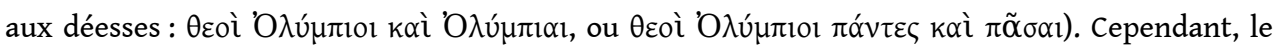
sens de ces ensembles devrait parfois être précisé (à Sparte, par exemple, cette dénomination renvoie aux Dioscures: IG V, 1, $\mathrm{n}^{\circ} 447,161-169$ de notre ère).

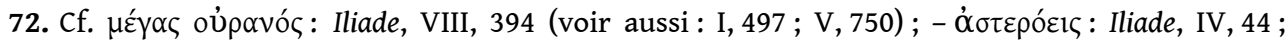
V, 769 ; VI, 108 ; XV, 371 ; XIX, 128, 130 ; Odyssée, IX, 527 ; XX, 113, etc. ; - عủpú : Iliade, III, 364 ; V, 867 ; VII, 178, 201 ; XV, 36, 192 ; XXI, 267, 272, 522 ; Odyssée, I, 67 ; IV, 378, 479 ; V, 169, 184, 303 ;

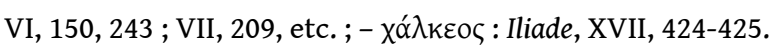

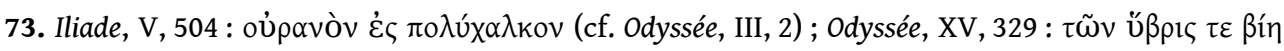

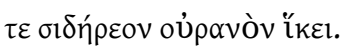

74. Clameur: Iliade, XII, 338 ; kleos : Odyssée, VIII, 73-74 (des hauts faits, chantés par l'aède) ; IX,

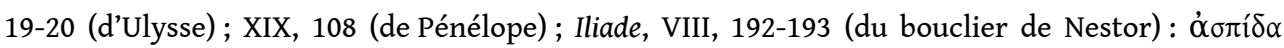

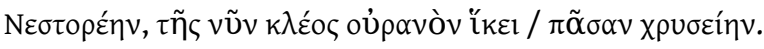

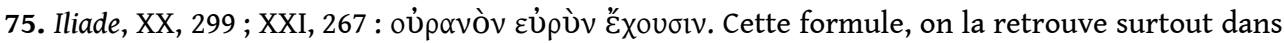
l'Odyssée : XI, 133 ; XVI, 183, 200, 211 ; XIX, 40 ; XXII, 39 ; XXIII, 280, etc. (voir aussi les références

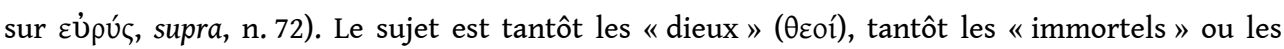

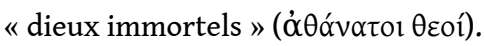

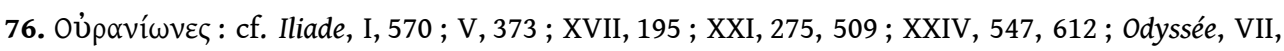
242 ; XIII, 41 ; غ̇ं̃oupávior : Iliade, VI, 129, etc. ; Odyssée, XVII, 484.

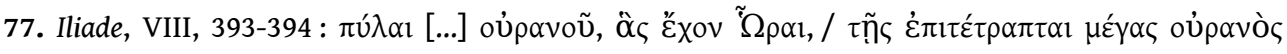

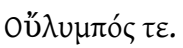

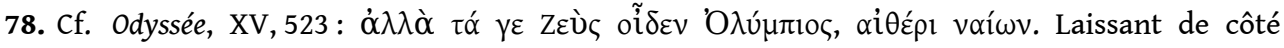

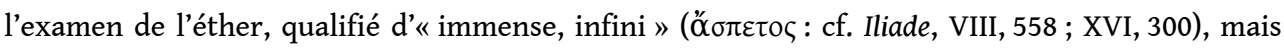

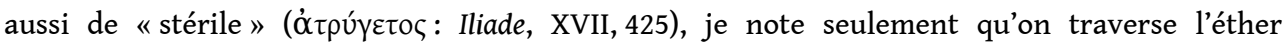

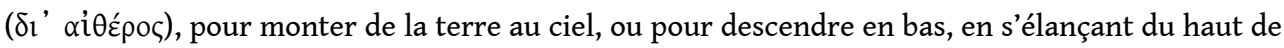
l'ouranos (cf. Iliade, II, 458 ; XIX, 350-351).

79. Athéna vient du ciel pour calmer la colère d'Achille (Iliade, I, 194-195: $\tilde{\eta} \lambda \theta \varepsilon \delta$ 'A A

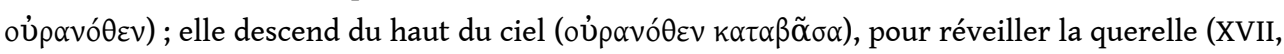
544-545), ou pour aider Ulysse (Odyssée, XX, 30-31). Mais tout immortel peut aussi descendre du

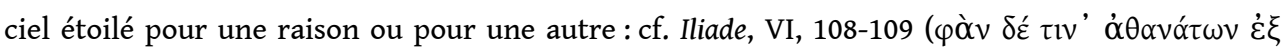

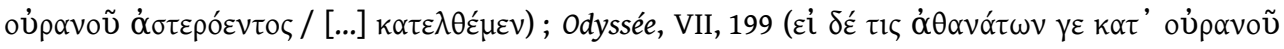

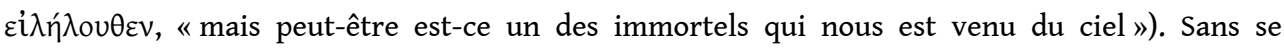
déplacer, Phoibos Apollon amène « du ciel sur la plaine » (oủ $\rho \alpha v o ́ \theta \varepsilon v \pi \varepsilon \delta$ เó$\theta \varepsilon v$ ) une nuée sombre 
pour protéger du soleil le corps d'Hector, trainé sur le sol par Achille : Iliade, XXIII, 188-191. Pour l'Olympe, voir supra, avec la n. 58.

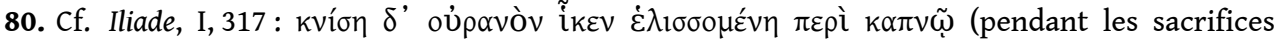
offerts à Apollon par les Achéens, « la knisê parvenait jusqu'au ciel, dans des spirales de fumée »);

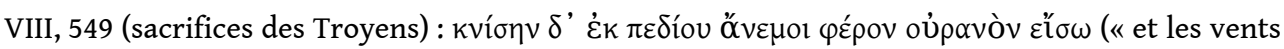
portaient la knisê de la plaine jusqu'au ciel »).

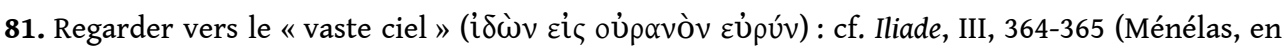

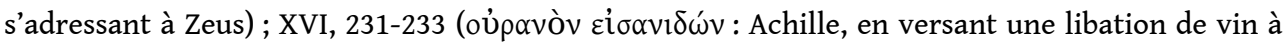
Zeus de Dodone) ; XXI, 272-272 (Achille, en gémissant, s'adresse à Zeus père). Tendre les mains

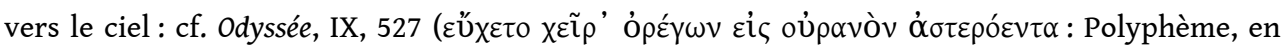
priant son père Poséidon). Les mains et les yeux : Iliade, VII, 177-179 (les Achéens, en levant les mains vers les dieux, tandis que chacun priait Zeus père, en regardant le ciel; cf. aussi v. 200-201).

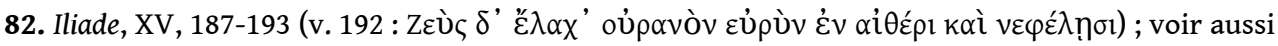
supra, avec la n. 44.

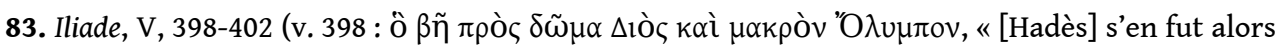
vers le palais de Zeus, et le haut Olympe »).

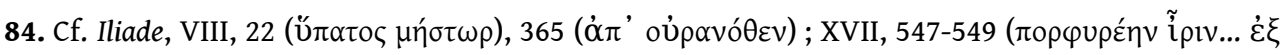

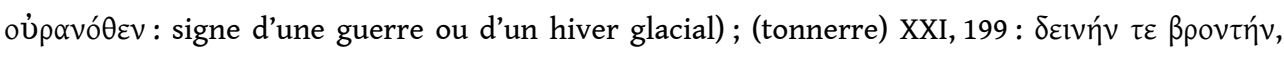

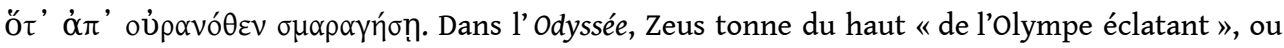

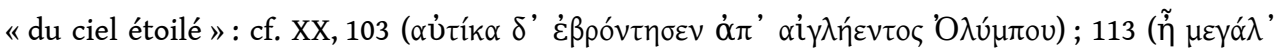

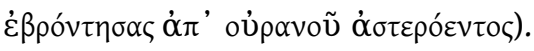

85. Pour lesquels il y a, entre autres, un excellent ouvrage collectif : voir Pironti \& Bonnet (2017).

\section{RÉSUMÉS}

Il existe une opposition très connue, maintes fois utilisée par plusieurs Modernes, qui classe les différentes puissances divines en deux groupes séparés, "olympiennes » (ou, plus rarement, «ouraniennes») et «chthoniennes». Parfois on fait même la distinction entre "religion olympienne » et « religion chthonienne », pour établir ainsi une sorte de dichotomie rigide entre un rituel adressé aux soi-disant «Olympiens/Ouraniens » et d'autres rites qui auraient concerné seulement des entités "chthoniennes", qu'il s'agisse de dieux ou de héros. En choisissant ce titre, plutôt général et neutre (Dieux d'en haut et d'en bas), ce texte vise à sortir de cette opposition tranchée, et à saisir la façon dont les Grecs eux-mêmes concevaient leurs dieux, en l'occurrence dans l'épopée homérique, en scrutant le vocabulaire, le sens des mots utilisés pour qualifier une divinité, un espace, un mode d'action.

A well-known opposition used by a number of scholars classifies the different divine powers into two distinct groups, the "Olympians" (occasionally known as the "Heavenly"), and the "Chthonians". Sometimes, a distinction is even drawn between "Olympian religion" and "Chthonian religion", in order to establish a kind of rigid dichotomy between rituals addressed to the so-called "Olympians" or "Heavenly" gods, and other rites concerning only "Chthonian" entities. This paper, under a more general and neutral title (Gods of the Above, Gods of the Below), aims to withdraw from this clear-cut opposition and to perceive how the Greeks themselves 
conceived their gods. The paper surveys the case of the Homeric epic, examining the vocabulary, and the sense of the words used to define a divinity, space, and modes of action.

\section{INDEX}

Keywords : “Olympian”/"Heavenly” gods, "Chthonian” gods, Homeric epic, chthôn/gaia, Zeus, Olympus, heaven

Mots-clés : dieux « olympiens/ouraniens », dieux « chthoniens », épopée homérique, chthôn/ gaia, Zeus, Olympe, ciel

\section{AUTEUR}

\section{STELLA GEORGOUDI}

École Pratique des Hautes Études, PSL, Unité de recherche ANHIMA, Paris stgeorg@ehess.fr 